\title{
Synaptic tuning
}

DOI:

10.1038/nrn1936

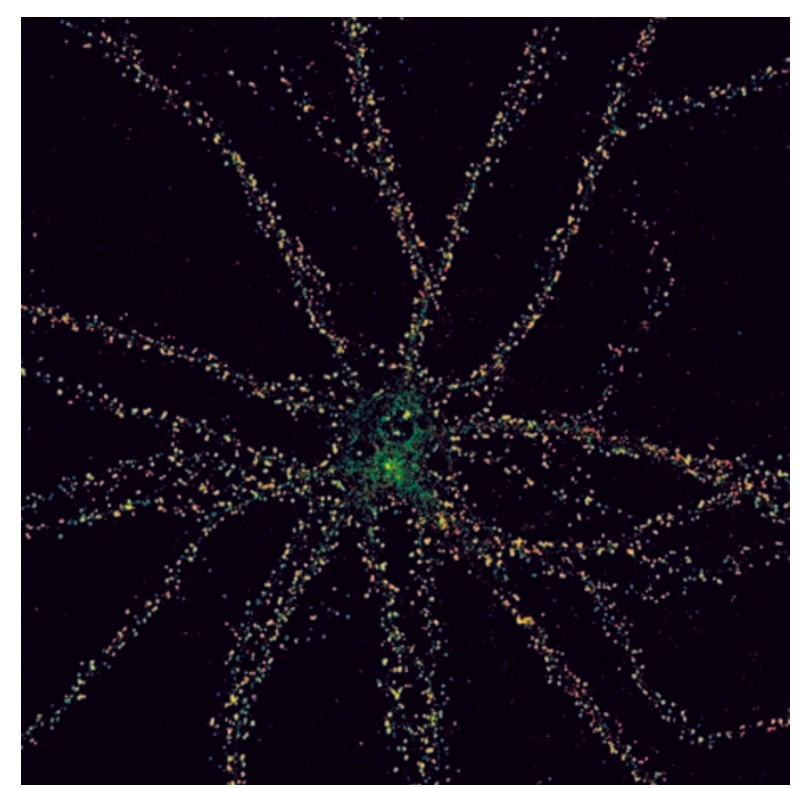

Dual labelling of NR2B subunit (green) and PSD-95 (red) following dopamine D1 receptor activation in striatal neurons at 21 days in vitro. Image courtesy of $A$.

Dunah, Harvard Medical School, USA

\begin{abstract}
Neurons in the striatum receive a number of convergent inputs from other regions, including glutamatergic inputs from the cortex and dopaminergic inputs from the substantia nigra. Interactions between these inputs are crucial for the acquisition of motor learning and regulation of movement: activation of dopamine D1 receptors exerts a strong modulatory effect on the strength of corticostriatal synapses by enhancing NMDA ( $N$-methyl-D-aspartate) receptor function. Reporting in the Journal of Neuroscience, Dunah and colleagues show that $\mathrm{D} 1$ receptor activation leads to the enhanced trafficking, clustering and membrane insertion of NR2B-subunit-containing NMDA receptors, and that this
\end{abstract}

process is mediated by tyrosine phosphorylation.

Treatment of striatal neurons with a D1 receptor agonist led to an increase in the dendritic staining intensity of NMDA receptor NR2B subunits. There was also an increase in the association of NR2B subunits with PSD-95 - a scaffold protein required for the assembly of NMDA receptors - and in the surface localization of NR2B-containing receptors. Trafficking and membrane insertion of NR1 subunits was also increased, but to a lesser extent. This finding is consistent with NR1 subunits being obligatory to the formation of functional NMDA receptors. There was no effect of the D1 agonist on NR2A subunit staining intensity, clustering or localization, and a dopamine D2 receptor agonist had no detectable effect on any NMDA receptor subunit.

Subunit phosphorylation is known to be an important regulatory mechanism of glutamate receptor function in the CNS: NR2 subunits are phosphorylated at tyrosine residues, and this phosphorylation is altered in models of synaptic plasticity and Parkinson's disease. The authors used quantitative morphometric analysis to determine whether NMDA receptor subunit targeting and localization is regulated by phosphorylation state. Treatment of striatal neurons with pervanadate, a tyrosine phosphatase inhibitor, led to elevated staining intensity of NR1, NR2A and NR2B subunits in neuronal dendrites, an increase in their association with PSD-95, and enhanced insertion of all three subunits into the plasma membrane.
Treatment of striatal neurons with genistein, a tyrosine kinase inhibitor, had the opposite effect. These findings show that tyrosine kinases and phosphatases exert a reciprocal effect on the trafficking, clustering and membrane insertion of these three subunits.

The authors went on to show that tyrosine phosphorylation is the mechanistic basis for regulation of NMDA receptors by dopamine. The effect of D1 receptor activation on NR2B trafficking was blocked by genistein, indicating that tyrosine phosphorylation is required. Using a phosphorylation site-specific antibody and fluorescence lifetime imaging microscopy (FLIM), the authors showed that D1 receptor activation leads to an increase in phosphorylation of NR2B at tyrosine residue 1472 , and that phosphorylation at this site strongly correlated with dendritic localization (as opposed to somal localization) of the subunit.

These results reveal a crucial role for tyrosine phosphorylation as a mechanism for dopamine-regulated trafficking of NMDA receptors in striatal neurons and, consequently, synaptic strength. Modification of this pathway might be a useful therapeutic target for Parkinson's disease and other disorders with abnormal NMDA receptor function. It will be of interest to determine the upstream modulators of targeting of other NMDA receptor subunits.

Daniel McGowan

ORIGINAL RESEARCH PAPER Hallett, P. et al. Dopamine $\mathrm{D}_{1}$ activation potentiates striatal NMDA receptors by tyrosine phosphorylationdependent subunit trafficking.J. Neurosci. 26, 4690-4700 (2006) 